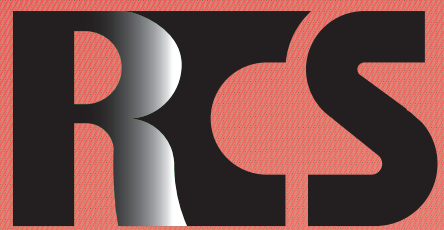

Depósito legal ppi $201502 Z U 4662$

Esta publicación científica en formato digital es continuidad de la revista impresa Depósito Legal: pp $197402 Z U 789$

- ISSN: 1315-9518 • ISSN-E: 2477-9431

Revista de Ciencias Sociales

Universidad del Zulia. Revista de la Facultad de Ciencias Económicas y Sociales Vol. XXVII. No. 2

Abril-Junio 2021

Esta publicación científica en formato digital es continuidad de la revista impresa Depósito Legal: pp $197402 Z$ Z789 ISSN: 1315-9518 


\title{
Formación por competencias del profesional en administración: Desde un enfoque contingencial
}

\author{
Ramos Farroñán, Emma Verónica* \\ Otero Gonzáles, Carlos Alberto** \\ Heredia Llatas, Flor Delicia*** \\ Sotomayor Nunura, Gioconda Del Socorro****
}

\section{Resumen}

El proceso de formación del profesional en administración egresado de las universidades en América Latina, debe fundamentarse en la estructuración de un perfil profesional, laboral y personal, con un alto grado de identificación sociocultural con el país, y acorde a las demandas globales. Por lo anterior, el objetivo de este estudio consistió en reflexionar sobre la formación profesional por competencias del administrador, desde un enfoque contingencial. Fue una investigación de tipo descriptiva-transversal, caracterizando las variables; de forma cualitativa, sin ser manipuladas; lo que resultó en un estudio no experimental, basado en la observación del fenómeno existente, analizado por medio de fuentes documentales versados en el tema de competencias profesionales y formación profesional de los administradores. Además, el estudio se apoyó en teorías como la del enfoque de contingencias y postulados de Bédard (2003). Entre los resultados se encontró que la formación profesional del administrador permite potenciar las capacidades de los estudiantes a nivel funcional, a pensar con sentido crítico e integral. Se concluyó que el proceso de formación de los administradores debe estar orientado a formar y egresar individuos capacitados para afrontar las problemáticas surgidas en las organizaciones, según los momentos y situaciones específicos del entorno, donde se encuentren ubicadas.

Palabras clave: Formación profesional por competencias; enfoque contingencial; rombo de Bedard; administración; pensamiento crítico.

\footnotetext{
Doctora en Ciencias de la Educación. Magister en Administración con mención en Gerencia Empresarial. Licenciada en Administración. Docente Tiempo Completo de la Universidad César Vallejo, Filial Piura, Perú. E-mail: eramosf@ ucv.edu.pe; $\underline{\text { eramosfa@gmail.com iD ORCID https://orcid.org/0000-0003-1755-7967 }}$

** Candidato a Doctor en Administración por la Universidad Nacional Pedro Ruiz Gallo. Magister en Administración. Docente de la Universidad Señor de Sipán, Chiclayo-Perú. E-mail: carlosotero@crece.uss.edu.pe; carlosog15@ gmail.com Scopus Author ID: 57201736864. iD ORCID: https://orcid.org/0000-0002-6335-9083

*** Maestra en Docencia y Gestión Educativa. Coordinadora del Programa de Maestría en Gestión Pública. Docente adscrita a la Escuela de Posgrado de la Universidad César Vallejo, filial Chiclayo, Perú. E-mail: fheredia@ucv.edu.pe (iD) ORCID: https://orcid.org/0000-0001-6260-9960

**** Doctora en Ciencias de la Educación. Docente de la Universidad Señor de Sipán, Chiclayo-Perú. E-mail: gioocondasn@crece.uss.edu.pe iD ORCID: https://orcid.org/0000-0001-6082-0893
} 


\title{
Training by competencies of the professional in administration: From a contingency approach
}

\begin{abstract}
The training process for management professionals graduated from universities in Latin America must be based on the structuring of a professional, work and personal profile, with a high degree of sociocultural identification with the country, and in accordance with global demands. Therefore, the objective of this study consisted of reflecting on the professional training by competencies of the administrator, from a contingency approach. It was a descriptive-cross-sectional investigation, characterizing the variables; qualitatively, without being manipulated; which resulted in a non-experimental study, based on the observation of the existing phenomenon, analyzed through documentary sources versed in the subject of professional skills and professional training of administrators. In addition, the study was supported by theories such as the contingency approach and postulates of Bédard (2003). Among the results, it was found that the professional training of the administrator allows to enhance the capacities of students at a functional level, to think critically and comprehensively. It was concluded that the training process for administrators should be aimed at training and graduating individuals trained to face the problems that arise in organizations, according to the specific moments and situations of the environment, where they are located.
\end{abstract}

Keywords: Vocational training by competencies; contingency approach; Bedard rhombus; administration; critical thinking.

\section{Introducción}

En la conferencia mundial sobre la Educación Superior, de la Organización de las Naciones Unidas para la Educación, la Ciencia y la Cultura (UNESCO, 2009), se reconoció que esta es, de responsabilidad pública, de todos los gobiernos; su contribución al desarrollo sostenible de las naciones, se percibirá en la medida en que las instituciones de enseñanza superior, conservando su autonomía y libertad académica, formen profesionales con pensamiento crítico e independiente en el campo de la investigación y la innovación, promoviendo el desarrollo del capital humano, las tecnologías de información y comunicación, la transferencia de conocimientos y resolución de problemas, de acuerdo a los requerimientos de la sociedad, dentro de las perspectivas de la internacionalización, regionalización y globalización.
Al respecto, se debe decir que durante décadas la educación superior y la formación universitaria en todos los ámbitos y modalidades; han enfrentado grandes desafíos y retos, donde se han hecho esfuerzos relevantes para la construcción de un proceso formativo de calidad, en vista de la responsabilidad para dar respuesta a las exigencias y a las demandas de una sociedad cada vez más necesitada de avances y desarrollo en ciencia tecnología e innovación (Companioni, 2015; GarbanzoVargas, 2016; Acevedo-Duque, et al., 2020). En este aspecto, Merino (2020), señala que:

Todo ello debe ejecutarse, con miras a ofrecer profesionales capacitados que respondan a los desafíos del campo laboral con eficiencia y cabalidad; para afrontar estos desafíos, la formación de un currículo profesional basado en competencias, es necesario y urge su inmediata aplicación en todas las carreras universitarias. (p.216)

En ese sentido, la educación superior 
tiene un impacto directo en la competitividad del país a través del efecto sobre la productividad y de la mano de obra que forma. Por lo anterior, la existencia o disponibilidad de profesionales competentes es, sin lugar a dudas, uno de los factores que analizan los inversionistas al tomar sus decisiones en la creación de empleos (Sistema Nacional de Evaluación, Acreditación y Certificación de la Calidad Educativa [SINEACE], 2012).

En la actualidad en el ámbito de la educación superior, se debe tender a superar muchos más retos, y establecer igualdad de condiciones para mayor accesibilidad, promover la vinculación con los grupos de interés (sociales, productivos y sectoriales), desarrollar talento humano idóneo, atraer y retener personal académico comprometido con la enseñanza y los procesos de investigación e innovación, así como también, a impulsar el desarrollo del pensamiento crítico e independiente; que promueva e incentive al deseo por aprender durante todas las etapas de la vida, tanto en el aspecto profesional como el personal.

Para tal fin, resulta imprescindible que las universidades innoven y mejoren los procesos de enseñanza aprendizaje, la didáctica, los métodos que emplean, a modos de permitir a los egresados, entrar en el campo de la competitividad de forma asertiva y efectiva, con resultados que se observen en su calidad profesional.

En este aspecto, es preponderante que dentro del sector universitario, se realicen cambios y actualizaciones curriculares que eleven y posicionen los programas educativos de las diferentes instituciones formativas, al nivel de las universidades en el ámbito internacional, igualándose lo más posible, tomando ventaja competitiva que provea a sus egresados de una formación académica óptima.

Considerando que la formación profesional de una persona, está vinculada a los estudios realizados y al grado académico obtenido, a la conjugación de un aprendizaje formal pero también a la autorrealización; de manera que inculcar a los estudiantes el hábito de pensar y analizar de forma crítica; supone potenciar la capacidad de poder crear, innovar y producir; así como afianzar la relación entre los recursos propios y los existentes en su entorno, en aras de conseguir un producto $\mathrm{y} / \mathrm{o}$ servicio más competitivo; en esto radica, las capacidades y competencias que deben poseer para satisfacer las necesidades y demandas de su ambiente laboral y del medio donde se encuentre inserto como profesional.

En cuanto al profesional en administración, Lombana, et al. (2014), estiman de gran importancia el desarrollo de las competencias genéricas y específicas para estos profesionales; las cuales comprenden las habilidades, aptitudes, capacidades, que conforman su perfil profesional.

Atendiendo a los contenidos y aspectos que se manejan en el análisis por competencias, la educación superior tiene un impacto directo en el área de la competitividad y productividad organizacional o empresarial, debido a la influencia que tiene sobre la efectividad y eficiencia de la mano de obra que está formando. La presencia de profesionales competentes, es sin lugar a dudas, uno de los factores que analizan los inversionistas y los empleadores al tomar sus decisiones (SINEACE, 2012).

La corriente seguida por McClelland en 1973, establece que las competencias se relacionan con las cualidades y características de un individuo para clasificar en una tarea o función específica, sobre todo en el ámbito educativo (Lombana, et al., 2014). Además de las competencias profesionales o específicas, las genéricas y personales, se deben tener en cuenta las demandas que la comunidad o entorno social efectúa, y que atendiendo a esto, se contribuya a elevar la calidad de la educación superior; en el sentido de construir un perfil que les permita el acceso al conocimiento y a la información, para una participación social plena, por medio de habilidades y destrezas con las cuales puedan intervenir efectivamente en la resolución de los problemas de su realidad inmediata.

Destaca aquí, la importancia de contar con personal académico, que estén 
a su vez capacitados para implementar procedimientos eficaces, recursos y estrategias adecuados, con el fin de formar profesionales altamente exitosos y competitivos laboralmente hablando; por ello, Cabrera, Cantelar y Valcárcel (2017), opina que “el desarrollo de competencias requiere de una retroalimentación constante del docente que lo lleva a cabo; los materiales principales empleados como medios de enseñanza deben ser aquellos que reflejen situaciones reales y experiencias en el desempeño" (p.289).

Lo antes descrito, dio pie a la formulación del objetivo de este estudio, el cual consistió en: Reflexionar sobre la formación profesional por competencias del administrador egresado de las universidades peruanas, desde un enfoque contingencial. Por medio de este estudio, se podrá confirmar que el proceso de formación de estos profesionales está intencionalmente orientado a formar y egresar ciudadanos aptos e idóneos para insertarse de forma exitosa en cualquier empresa u organización donde se les requiera (Ramos, 2016; Rabanal, et al., 2020).

Este es un tipo de investigación descriptiva-transversal, con la cual se trató de especificar los aspectos característicos de las variables del estudio por medio de la información disponible; es una indagación cualitativa, con la cual no se pretendió generalizar resultados de manera probabilística (Hernández, Fernández y Baptista, 2014). Es un estudio no experimental, basado en la observación de fenómenos existentes en la realidad, los cuales podrán ser sometidos a un estudio más profundo dada las características del hecho observado, el cual puede ser medido conforme a las expectativas que se creen con esta investigación en torno a las competencias profesionales que se exigen en los ámbitos laborales, donde existen perfiles profesionales establecidos para que los profesionales en administración puedan ocupar algún puesto en las organizaciones, tomando en cuenta el entorno, sea este regional o internacional.

De igual manera, puesto que no se han manipulado las variables su diseño es no experimental; los datos e información manejada son provenientes de fuentes secundarias, bibliográficas y artículos especializados en el tema (Hernández, et al., 2014)

\section{Conceptualización de competencias profesionales}

El cambio es la constante hoy en el mundo, por esto las empresas, las organizaciones y las personas están llamadas a transformarse en forma integral y permanente. La Universidad, como institución forjadora de procesos formativos, es el órgano encargado de mantener, desarrollar y promover esta preparación integral, así el desarrollo de estos procesos se orienta a una verdadera transformación humana, que emerge para hacer de la formación académica, un servicio pertinente con las demandas contextualizadas de la sociedad.

Al mismo tiempo, esta realidad demanda a los egresados de las universidades una formación que responda ante los retos actuales, y que posea una preparación integral para afrontar el cambio y la incertidumbre, a la vez que se hagan competentes para corresponder con la dinámica organizacional y social. Lo antes planteado, ha convertido este aspecto de las competencias, en objeto de continua discusión, debido a las concepciones teóricas y técnicas, que llevan a la diatriba de, si deben ser tomadas como parámetro o lineamiento para la construcción del perfil de los profesionales, o dejar su adquisición a través de la práctica laboral. Al respecto, se muestra un cuadro de conceptualizaciones sobre competencias que varios autores han propuesto basados en sus experiencias y desde contextos diferentes, de forma tal, que puedan establecerse similitudes y comparaciones entre las mismas (ver Cuadro 1). 


\section{Cuadro 1 \\ Conceptualizaciones sobre competencias}

Autores Conceptualizaciones de Competencias

Burns y Klingstedt (1973)

Schroder (1989)

Management Charter Initiative (1991)

Aledo (1995)

McLagan (1997)

Llopart (1997)

Levy (1997)

Chomsky (2014)

Alles (2008)

Especialista en Tecnología Educativa, esta concepción fue manejada desde una perspectiva conductista, enfocada en la formulación de objetivos que las constituían. El concepto se profundizó por la idea de "competencias mínimas" (minimal competency); y fue aplicado al campo de la formación técnica y luego a la enseñanza de idiomas.

Define la actuación de competencia como actuación - un conjunto de comportamientos relativamente estables, que van a producir una mejor adecuación en los equipos de trabajo, en ambientes organizacionales complejos.

Las nombran como competencias ocupacionales y son aquellas habilidades para realizar actividades propias de una ocupación, o función según el nivel de actuación esperado.

Las definió como un conjunto específico de conductas observables evaluables y clasificadas- son categorías de conductas.

Señala que existen diferencias entre el significado de competencias, tanto si se refiere a las organizaciones como a los individuos; además de las ventajas e inconvenientes presentes en cada aproximación conceptual. Según este autor, el significado de competencias viene dado por los siguientes elementos:

- $\quad$ Competencias como tareas- tareas de trabajo y actividades.

- $\quad$ Competencias como resultado- estas conciben que la habilidad produce beneficios a la empresa.

Constituyen un conjunto de características personales, y conocimientos que confieren a las personas capacidades para desempeñar las funciones correspondientes a su ocupación, de forma satisfactoria y acorde con los objetivos y estrategias organizacionales.

Para esta autora, las competencias representan la unión entre las características individuales y las cualidades requeridas para llevar a cabo misiones profesionales. Son el conjunto de conductas organizadas y relativamente estables y movibles para cuando sea preciso.

Se refiere a estas como algunos aspectos del conocimiento y a las habilidades, necesarias para lograr resultados y cumplir las exigencias de una situación específica; siendo estas capacidades reales para el logro de objetivos.

Relaciona el talento con las competencias; refiriéndose a estas últimas como las características de personalidad, comportamientos que generan un desempeño exitoso en un puesto de trabajo. Son las competencias las que producen o permiten el desempeño superior de una persona.

Fuente: Elaboración propia, 2021

En este punto cabe destacar, que el tema sobre las competencias, como se observa, ha sido abordado por diferentes autores, quienes desde diferentes perspectivas y marcos teóricos referenciales, han realizado aproximaciones sobre su definición de acuerdo al contexto que han manejado. En ese sentido, Zabala, Marcano y Chávez (2013), infieren que:

En la actualidad, existen diversos enfoques explicativos de las competencias dentro de la dinámica social y ocupacional, que las asume con su condición humana como las capacidades para el desempeño exitoso de una ocupación, tendientes a la integración para llegar a la integralidad. (p.162)
En el mismo orden de ideas, Castañeda (2015), explica que:

Las competencias no sólo deben basarse en conocimientos y habilidades que parten de la formación profesional específica, también se derivan de la capacidad para saber resolver un problema con base en las habilidades y destrezas obtenidas a través de la educación, que nos permite desarrollarnos profesionalmente, es decir, la capacidad de responder a demandas complejas. (p.253)

Luego de revisar las diferentes conceptualizaciones realizadas por varios autores y estudiosos del tema; se puede 
inferir que las competencias profesionales son cualidades que deben poseer los profesionales o egresados universitarios, las cuales están determinadas tanto por destrezas y habilidades adquiridas o desarrolladas durante la formación profesional específicas de cada carrera, como aquellas cualidades o características personales que posee el individuo y ha ido fortaleciendo con la madurez intelectual y emocional, (como ser social y cultural), todo lo cual va a servirle para resolver situaciones problemáticas en el ámbito profesional, del mismo modo que influyen en sus relaciones interpersonales, dentro o fuera de su entorno laboral.

\section{Formación profesional por competencias del administrador}

Es importante mencionar, en relación a la función de las competencias en el contexto de la formación profesional, que "la educación por competencias surge desde finales del siglo XX en Europa, con el Proyecto Tuning, impulsado por la Unión Europea y posteriormente por el proyecto DeSeCo (Definición y Selección de Competencias), (...), que promueve la Organización para la Cooperación y el Desarrollo Económicos (OCDE)" (Castañeda, 2015, p.255).

De igual forma, el Proyecto Tunning, creado para América Latina en 2004, cuyo propósito fue mejorar las condiciones del aprendizaje, por medio de la creación y clasificación de los perfiles de los egresados, a modo de facilitar la movilidad de estos como profesionales; siendo la razón de ser del proyecto, que este se fundamentó en la búsqueda de puntos de referencia comunes, centrándose en las competencias y en las destrezas basadas en el conocimiento (Del Alcázar, 2020).

Por lo tanto, su definición de competencias, está enmarcada bajo la denominación de "competencias universitarias"; las cuales representan el enfoque en las habilidades prácticas accionables en áreas instrumentales, interpersonales y sistemáticas, que deben desarrollar los estudiantes durante la formación profesional en sus carreras; de forma transversal e independientes de las específicas de la profesión o asignatura que cursen (Del Alcázar, 2020).

Por su parte, Cejas, et al. (2019) definen la formación por competencias como: “(...) un proceso de enseñanza y aprendizaje que está orientado a que las personas adquieran habilidades, conocimientos y destrezas empleando procedimientos o actitudes necesarias para mejorar su desempeño y alcanzar los fines de la organización y/o institución" (p.95).

En ese aspecto, Casanova, et al. (2018); y Jiménez-Silva, et al. (2019), opinan que en la formación universitaria los contenidos curriculares deben fortalecer la capacidad cognitiva del estudiante para así reconocer las oportunidades, forjar conocimientos y tecnologías que contribuyan a la resolución de problemas así como inciten a alcanzar una mejor calidad de vida. Destacando, que el enfoque de competencias, tal como lo presentan Useche y Artigas (2019):

(...) debe adaptase a la necesidad de cambio, omnipresente en la sociedad internacional, bajo una multiplicidad de formas. Por lo que, la competencia laboral es dinámica e imprime énfasis y valor a la capacidad humana para innovar, anticipar modificaciones y prepararse a éstas, en vez de convertirse en víctima pasiva, arrasada por transformaciones sin control. (p.387)

Así mismo, la formación académica profesional ha de ser contextualizada, además contar con recursos humanos preparados y comprometidos con el proceso formativo, para el logro de la excelencia y pertinencia con las demandas sociales; tal como lo señalan, Cabrera, et al. (2017) "el docente, como facilitador del proceso de aprendizaje, debe resignificar su función hacia un papel más asociado con la conducción del aprendizaje" (p.992).

Al respecto, las universidades juegan un rol protagónico en la formación del individuo, al proveer la adquisición de habilidades basadas en la generación, difusión y uso del conocimiento, que les permitan 
responder a las demandas del mercado, así como proyectar y predecir ante escenarios alternativos o adversos. Debe hacerse, bajo una dinámica determinada por la capacidad de interacción y consolidación de competencias, que a la vez fortalezcan la capacidad científica e incrementen la productividad así como la competitividad de las empresas u organizaciones.

En el proceso de formación profesional por competencias, existe transmisión de información, entrenamiento o capacitación de una persona o un grupo, lo que les permitirá aprender a realizar una actividad con características específicas, y a desempeñar esa tarea o función eficientemente $\mathrm{y}$ satisfactoriamente en un área laboral concreta (Tobón, 2008).

De lo anterior se desprende que, en la actualidad las empresas $\mathrm{u}$ organizaciones tengan en cuenta características específicas sobre las funciones o cargos a desempeñar, con respecto al perfil que deben poseer los posibles empleados o candidatos a un puesto, para ser elegibles. Todo lo cual, ha ocasionado que en las universidades se repiensen los currículos de las carreras que se ofrecen, y se atiendan estas exigencias, promoviendo la formación profesional para el desarrollo de competencias profesionales y personales. Icarte y Labate (2016), infieren que, "el desafío para las universidades en este marco, reside en rediseñar sus matrices formativas alrededor de las competencias de salida más que alrededor de las tradicionales asignaturas" (p.5).

Las demandas o exigencias por parte de las organizaciones, empresas o industrias, están enfocadas a una mayor preparación o formación profesional del egresado en áreas diversas y no solo en las específicas de cada carrera, esto conlleva a tomar como principal rasgo para emplear individuos, el nivel de la calidad de dicha formación; en concordancia con la naturaleza y políticas organizacionales, en pro de beneficios para el entorno social, por ello Cabrera, et al. (2017) afirman que:

Los métodos, técnicas y procedimientos empleados y aplicados en el proceso de formación, favorecen el desarrollo de las competencias profesionales, por los profesores de una carrera universitaria, en tanto que activan el proceso de formación y permiten que cada individuo trace su estrategia de autoaprendizaje a favor de las necesidades que se le planteen durante la ejecución de cada programa y plan de estudios. (p.992)

Ahora bien, los procesos de formación de profesionales en las universidades, orientados al desarrollo de la calidad académica, contribuyen para hacer de la educación un servicio más pertinente con las demandas sociales; donde adquieren un significado predominante: El saber, así como el hacer y el ser. Al desarrollar estos aspectos en un proceso de aprendizaje por competencias centrado en el estudiante y su incidencia en el contexto social; ofrece aprendizajes o conocimientos, socialmente significativos; que habilitan a estos profesionales para operar con eficacia en contextos específicos, respondiendo a cabalidad, ante las dificultades, situaciones y retos propios de la época y del entorno.

Según Useche y Artígas (2019), el egresado de un proceso de formación por competencias, posee "una compleja estructura de atributos necesarios para el desempeño en situaciones adversas donde se combinan conocimientos, actitudes y habilidades en las tareas a desempeñar en determinadas situaciones" (p.386).

Lo anterior aplica en el sentido que, en un proceso de formación por competencias no se proponen aprendizajes fragmentados, actitudes, destrezas y conocimientos aislados, que se suman sin articularse entre sí; al hablar de formación por competencias en la formación profesional, se refiere a una combinación de conocimientos, habilidades, comportamientos así como actitudes, formando una estructura, que se demuestran en un contexto determinado y que se pueden transferir en diferentes condiciones, a través de la actualización permanente. Por lo antes dicho, un profesional competente, según expone, Ramos (2016):

a. Es aquel que se apropia de las teorías, las leyes, los conceptos, las definiciones de la rama del saber en las cuales se desenvuelve, 
para poder actuar responsablemente y con posibilidades de hacer análisis, reflexiones, inferencias, inducciones, deducciones. Es hábil en su desempeño, mostrando destrezas, tanto orales y comunicativas como manuales, físicas y motoras.

b. Se empeña por hacer las cosas bien, porque está totalmente motivado con su profesión, porque lo hace conscientemente, demostrando en su desempeño profesional la convicción que tiene de la importancia social de su labor, con lo cual contribuye al desarrollo del país.

c. Demuestra haberse apropiado de los aspectos más significativos, que identifican la ética de su profesión. Trasciende sus propios aprendizajes, siendo capaz de potenciar sus recursos personales y dar soluciones creativas e innovadoras a nuevos problemas profesionales-coyunturales.

d. Es quien tiene la posibilidad de hacer transferencias de contenidos ya dominados hacia otros nuevos, por medio del autoaprendizaje y del adecuado manejo de la información.

Tomando en cuenta que el perfil específico de los egresados en Administración, como de cualquier otra carrera o profesión de las ciencias sociales u otras áreas, está conformado por las habilidades individuales, por las adquiridas con la práctica y los conocimientos aprehendidos por medio de los procesos formativos en las universidades, pero también a lo largo de toda su vida como estudiante y ser social; pudiéndose afirmar que existe una relación muy estrecha entre educación y competencias en el contexto de la educación superior; cuyo objetivo es fomentar las competencias; por lo tanto, los cambios del currículo universitario en busca de una integralidad en el profesional, se fundamentan en la necesidad de ofrecer una formación para la vida.

En la práctica formativa real, las competencias se forman en varias unidades del curso y son evaluadas en diferentes etapas; pueden estar divididas en competencias relacionadas con un área de conocimiento -o específicas de un campo de estudio-, y por competencias genéricas, que suelen ser comunes para diferentes cursos, y carreras (Lombana, et al., 2014.).

De acuerdo a esto, hay aspectos que intervienen en la discusión del proceso de formación del profesional en administración, que traen a colación la relación entre la teoría y la práctica; ante esto, algunos autores explican que el proceso de formación en la carrera de administración, ha sido objeto de diversos debates, en torno al énfasis en la teoría y en la práctica (Marín-Idagarra, 2013). Así que, las universidades deben direccionar los procesos formativos en la carrera de Administración con pertinencia y efectividad; promoviendo la apropiación del conocimiento en estos profesionales, con capacidad de reflexión y análisis para la solución de problemas cotidianos en las organizaciones, con un enfoque integral.

\section{La formación profesional del administrador bajo el enfoque contingencial}

El enfoque de contingencias, es una de las teorías contemporáneas de la administración, por medio del cual se analizan y consideran las circunstancias externas a las organizaciones, por lo que también se le llama enfoque situacional. A través de esta teoría, se deja claro que todas las organizaciones no son ni funcionan igual; lo que implica que su estructura y acciones tampoco lo sean; por lo tanto, se presentan diferentes situaciones o contingencias que se abordan y atienden de forma diferente (Segredo, 2016).

Por lo antes expuesto, es de gran importancia la formación profesional por competencias, en el caso del profesional en administración está directamente relacionado al planteamiento que se hacen muchos teóricos de la administración y la gerencia, quienes con frecuencia, no toman en cuenta la influencia del entorno en las diferentes dimensiones del ámbito organizacional y de las empresas; lo que hace presumir, que dichos enfoques (todos 
ellos) puedan ser compatibles con cualquier situación, tipo de organización, momento o circunstancia (Lara, Rodríguez y Del Canto, 2016), sosteniendo los precitados autores que:

El enfoque de contingencia establece que los dirigentes deberían diseñar las organizaciones, definir objetivos y reformular las políticas y planes de acuerdo a las condiciones predominantes del entorno, y las políticas y planes deben responder a los cambios de tales condiciones. Adicionalmente indican, que la gerencia debería tener las habilidades necesarias para anticipar, entender los cambios del entorno, y dar lugar al cambio dentro de la organización. (Lara, et al., 2016, p.74)

Al analizar la esencia de este planteamiento, y tomar en consideración lo que establece el enfoque situacional o contingencial, se observa que cuando se habla de la formación del administrador desde el punto de vista contingencial; se está atendiendo a una realidad y es que la gerencia es y debe ser situacional (Lara, et al, 2016). De igual forma, la formación profesional que se imparte en las universidades debe dar respuesta y concordar con las realidades existentes en el plano laboral y el ámbito real de las organizaciones; todo lo cual, incide en tener un mayor entendimiento y visión del tipo de competencias profesionales que se requieren.

Por lo anterior, también es conveniente describir algunos principios que rigen esta teoría o enfoque contingencial, de acuerdo con García (2020):

1. Los principios que rigen en una organización son situacionales, no son universales. Las situaciones del entorno lo definirán 2. El tipo de administración dependerá de la complejidad de cada situación. 3. Se otorga una visión de la capacidad que tiene la empresa para adaptarse a factores externos e internos, integrando ambos de la mejor manera 4. Se aplica un enfoque "Si...entonces", El "si" es un factor independiente, y el entonces será la variable dependiente, que será la técnica para implementar en dicha situación. (p.3)
Según lo anterior, se cree necesario tomar en cuenta los conceptos que la teoría contingencial contiene, en vista de, como su mismo nombre lo dice, esta se basa en las condiciones o contingencias que se desencadenan en las organizaciones; lo que establece, en la mayoría de casos, que las competencias profesionales del administrador, deben estar signadas por las pautas de los perfiles establecidos a nivel general, lo cual es el deber ser en todas las organizaciones (García, 2020). En ese sentido, el enfoque contingencial debe estar directamente relacionado con el perfil del profesional en administración y específicamente a las competencias que debe haber adquirido y desarrollado durante su carrera. Este aspecto puede palparse por el tipo de conocimientos que hayan adquirido, los cuales deben definir un perfil laboral, profesional y personal idóneo.

El mundo de la educación, al igual que de la formación profesional integral y en particular los profesores, no pueden ser ajenos a estos retos y desafíos (Sistema Nacional de Formación para el Trabajo [SENA], 2003). En este hecho, tiene que ver mucho la participación del personal académico y profesorado así como los aportes que realicen, durante el proceso formativo en sus roles de instructores y docentes.

Debe resaltarse aquí, que el personal docente de cualquier carrera es multidisciplinario, lo que le da características especiales al proceso de enseñanza; en la profesionalización de los administradores, convergen dos puntos de vista sobre este proceso; la concepción que los docentes tengan sobre el concepto de organización y la problemática de la administración y como entienden esta dinámica y su rol frente al de los estudiantes como futuros administradores, donde también son de relevante importancia las cualidades y perfiles profesionales de los formadores.

Por lo antes dicho, en la actualidad parte de la problemática existente sobre el tipo de instrucción que se da en las escuelas de administración, reside en la poca integración de los profesores y docentes, en los nuevos 
paradigmas organizacionales (Hernández, 2018).

Es necesario tener en cuenta que el tipo de conocimientos que se transmiten en esta área profesional, se basa en la "funcionalidad" con una perspectiva "racionalista e instrumentalista", muchas veces no existe la articulación que se requiere entre los conocimientos (del sub-campo específico) y los conocimientos e intereses de los estudiantes (Hernández, 2018), razón por la cual, existe una visión fragmentada sobre el tema de las organizaciones, sus fundamentos y temáticas relacionadas; en lo que también, influyen el estilo y modelos de enseñanza que se ejecutan, la cultura y las perspectivas para transmitir los contenidos (Hernández, 2018).

\section{Competencias profesionales del administrador y la aplicación del "rombo" de Bedard.}

En el mismo orden de ideas, en relación al análisis sobre el tipo de formación que están recibiendo los estudiantes de administración en las universidades y que tiene que ver con las competencias que deben construir y adquirir durante este período de su carrera profesional, está enmarcado desde el punto de vista de una de las teorías, por medio de la cual se ha estudiado, determinado y organizado la forma de interpretar al individuo en torno a sus caracterización profesional o laboral. Esta es la expuesta por Bédard, en 2003, quien consideró para su análisis 4 dimensiones filosóficas, conformada por 4 disciplinas: La praxeología (para las prácticas y la conducta), la epistemología (para los procedimientos y los métodos), la axiología (para los valores) y la ontología (para los principios y las teorías generales); de forma tal, que en cada dimensión se describen las competencias, o características que se pueden encontrar, tanto en el profesional, como para el individuo en lo personal o el ser.

El resultado basado en las experiencias personales y profesionales de Bedard (2003), le ha permitido denominarlo como "el rombo", esquema que se corresponde perfectamente con lo que se establece en el modelo con enfoque contingencial sobre la actuación y las relaciones del profesional en administración, pero además complementa perfectamente todo lo anteriormente expuesto sobre las competencias profesionales que deben poseer (Lombana, et al., 2014).

En consecuencia, en esta teoría denominada "el rombo"; se propone un esquema que permite identificar 4 tipos de competencias observables en un gerente: 1 . Las competencias del saber-hacer gerencia; 2. Las competencias del saber, propias de la dimensión epistemológica y del conocimiento administrativo; 3. Las competencias de la conducta ética y moral de los gerentes; y, 4. Las competencias ontológicas del ser. (Lombana, et al., 2014).

También se observa la interrelación de las 4 dimensiones filosóficas, las cuales mantienen una interconexión, es decir, son 4 aspectos particulares de una sola realidad; que permite percibir el hecho social en su totalidad y su relación con el enfoque de contingencias aplicado en muchas organizaciones, que a su vez se complementa con el tipo de formación profesional que se requiere para los futuros egresados en administración.

En ese sentido, la Ontología es la disciplina que condiciona y fundamenta a las otras tres; la epistemología y la axiología, trabajan cada una según sus funciones y fundamentaciones para conformar la totalidad del sistema. Las 4 están íntimamente articuladas, formando una interacción dialéctica y armoniosa; siendo importante destacar, que de presentarse un desacuerdo entre alguna de estas dimensiones, se crearían dificultades en su funcionamiento total o general; en la Figura I, se observa esta interacción, donde la praxeología, es la dimensión que justifica la existencia de la función administrativa. 


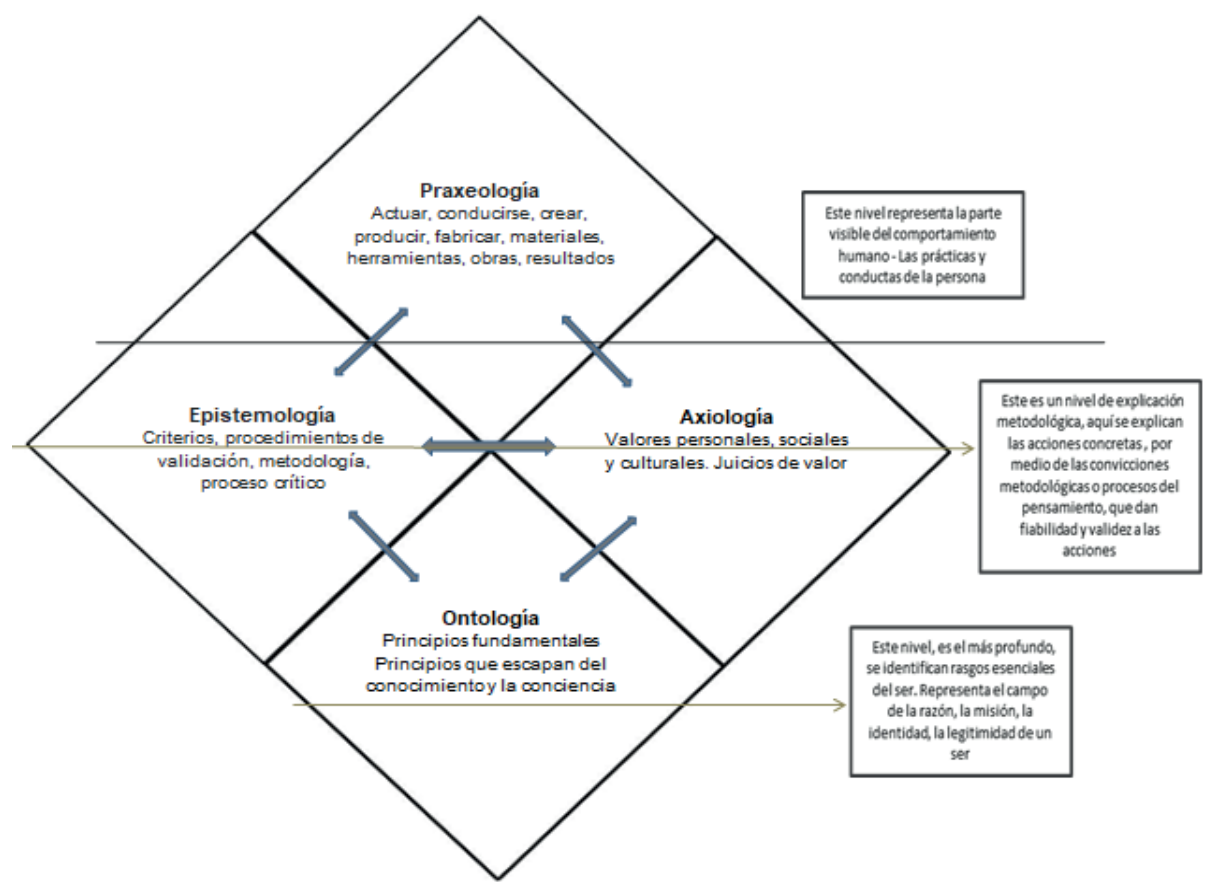

Fuente: Elaboración propia, 2021, basado en Bedard (2003), y Noreña (2018).

Figura I: Interrelación de las 4 dimensiones filosóficas

Para Bedard (2003), este conocimiento, "el rombo" se puede utilizar para armar una estructura interna, sobre una actividad concreta, o caracterizar la conducta de los individuos. "Este conocimiento profundo hace posible, que se haga una toma de conciencia de los elementos subyacentes a una práctica; y si es necesario puede guiar una intervención, con miras a modificarla o reforzarla" (p.85).

Interpretando, parte de la exposición realizada por Bédard (2003), esta gira alrededor de las siguientes ideas: Interesa para comprender las conductas de las personas, siendo oportuno conocer los fundamentos implícitos; prestar atención a acciones concretas, actitudes y palabras, así como "buscar donde están apoyadas; más allá de explicaciones racionales o lógicas” (p.82).

En su teoría, muy creativamente compara la figura del rombo con la de un iceberg, donde la parte que se ve fuera del agua no corresponde ni a la 8va parte del total del tamaño del iceberg (Bedard, 2003). Esta comparación refiere la complejidad de las características individuales, y humanas, en este caso, implementar el rombo sirve para identificar, distinguir y clasificar las cualidades o características que poseen los individuos, o profesionales, las cuales no pueden estar separadas y conforman una totalidad del producto que se va generando con el proceso formativo (integral), de una manera integrada.

En este orden de ideas, Zwerg-Villegas (2011), opina que en la actualidad en las 
escuelas de administración, se han basado más en las dimensiones funcionales para llevar a cabo el proceso de formación profesional del futuro administrador; para lo cual debe plantearse una reflexión sobre cómo debe ser la formación de estos profesionales, con un concepto de integralidad.

De igual manera, en vista que las escuelas de administración se manejan desde enfoques muy funcionalistas y no desde enfoques filosóficos, al momento de estructurar los procesos formativos de los futuros administradores, caracterizar a estos profesionales con un perfil específico, pero al mismo tiempo general en base a competencias, podría plantearse desde el punto de vista filosófico que esboza Bedard (2003) a través del "rombo" creado por ella. Esto implica, establecer las concepciones de cada dimensión y por medio de estas, realizar el análisis individual deseado o idóneo de cada profesional (perfil del egresado), que encierra 4 aspectos filosóficos fundamentales: La praxeología, epistemología, axiología y la ontología (Noreña, 2018).

El anterior "rombo" en conjunto con la teoría que encierra, es una herramienta muy útil para la consolidación de perfil por competencias del estudiante de administración; puesto que engloba de forma holística y armónica todas las características o cualidades de un individuo, tanto en lo profesional como en lo personal. Es necesario resaltar, que las competencias que conforman el perfil profesional de los administradores, se determinarán según el consenso entre los criterios de los académicos, los de los empleadores y los criterios propios de los profesionales que han cumplido con todos los requisitos para el término de su carrera (egresados); además de ello, será imprescindible contar con la información emitida por parte de las organizaciones, empresas y sitios de trabajo, quienes son los encargados de establecer los criterios que determinan el perfil del puesto (Merino, 2020).

\section{Conclusiones}

De este estudio se concluyó, que la formación profesional del administrador se concibe como un proceso continuo, que permite potenciar las capacidades de los estudiantes a nivel funcional, lo que es aceptable puesto que prepara al estudiante para la práctica e instrumentación de herramientas, con las cuales puede demostrar sus habilidades, destrezas y conocimientos adquiridos, al momento de interactuar en el ámbito laboral.

De manera que, inculcar a los estudiantes a pensar bien o mejor, con un sentido crítico e integralidad en sus análisis, esto implica potenciar la capacidad de innovación y productividad, así como afianzar la alianza con los recursos propios (competencias, habilidades, destrezas, y conocimientos adquiridos), en aras de conseguir un producto y/o servicio más competitivo, de mayor calidad y que satisfaga las necesidades prioritarias de la sociedad, a la par con el fin último y objetivos institucionales y académicos.

La formación por competencias, pero además contextualizada, les permite a los futuros egresados tener una visión detallada o específica de sus funciones y roles a desempeñar; lo que además, le ayuda a definir con claridad metas y objetivos, por tener una visión clara del contexto, de la localidad o región. Los programas y guías docentes empleados para la formación de un profesional competente, conlleva a la estructuración de los contenidos con miras a la transformación, hacia la excelencia en el desempeño laboral; manifestado y probado en las prácticas que desarrolla como estudiante, en interacción con su contexto sociocultural y laboral.

En ese sentido, se requiere de una formación profesional donde exista la construcción teórica-práctica por medio de una formación por competencias y además estructurada; esto puede ser posible, por ejemplo según lo planteado en este estudio, a partir de los aportes de la teoría sobre "el rombo", lo que permite una organización lógica pero filosófica de estos aprendizajes.

En el cumplimiento de los fines de las instituciones universitarias, en pro de la mejora de los procesos educativos, deben esforzarse para la articulación de los propósitos 
académicos de los docentes y profesores, en conjunción con los intereses de los estudiantes y futuros egresados. En vista de que, existen vacíos que dividen ambos grupos, debido a las deficiencias presentes durante el proceso formativo; ocasionado por la variedad de estilos instruccionales, los cuales no se basan en competencias.

Se hace necesario que los docentes en la carrera de administración, se vinculen con la naturaleza de la profesión (docentes), con miras a fortalecer sus capacidades y potencialidades, en correspondencia con los fines de la carrera donde llevan a cabo la función de formar. La formación por competencias, les exige contextualizar los contenidos programáticos, con una lógica integradora, que permita la fusión de los aspectos particulares y específicos del currículo, con los aspectos relacionados a la sub-área que imparte. Esto conlleva a corregir las deficiencias existentes, en los métodos aplicados, en conjunto con los responsables y corresponsables, de velar por la calidad en la formación profesional de los futuros administradores.

En este mismo orden de ideas, deben ponerse en práctica herramientas existentes, y recursos ya empleados por otros sectores, que están trabajando para darle un vuelco a la realidad existente, como lo han hecho desde el Proyecto Tuning Latinoamérica, en pro de la caracterización contextualizada, del perfil profesional de los administradores.

Todo lo anterior, se corresponde con lo que plantea el enfoque contingencial, que se aplica en las organizaciones, el cual amerita una preparación integral, basado en competencias, lo que sería el perfil idóneo. Es a partir de estas premisas que estos profesionales, podrán responder adecuadamente a las exigencias laborales actuales; incursionando eficientemente en las diferentes situaciones y contextos de la realidad laboral y social de su entorno próximo e internacional con una visión global.

No se quiere decir que se deba globalizar sobre el hecho del tipo de formación profesional de los estudiantes de administración; por el contrario, se requiere que estos se formen de forma autodidacta, en el sentido estricto de la autoformación, como bastión para aprovechar al máximo sus potencialidades, en concordancia con el medio donde se desenvolverán como profesionales.

Por lo tanto, los estudios previos al grado profesional deben estar enmarcados, en lo social y lo cultural de sus propios países, y luego sus especializaciones, propondrían diferentes opciones que complementen con nociones relacionadas con el ámbito externo, bien sea regional o del país.

Dicho lo anterior, el enfoque de contingencias, pudiera ser expuesto y relacionado con intencionalidad en el propio hecho de la formación académica de los administradores; promoviendo la amplitud de competencias por medio de la innovación y adaptación de la malla curricular, a las diversas situaciones organizacionales existentes, adecuando el perfil del egresado a las demandas laborales del mercado; pero a las sociales y culturales de igual manera.

Siendo urgente por parte de los entes responsables, actuar sobre la marcha y proceder a ejecutar revisiones periódicas, sí o sí, de los currículos, adaptándolos a los cambios, que también son continuos, de modo que se pueda contar con egresados, cuyos perfiles profesionales observados, estén dotados por competencias específicas, cada vez más especializadas, innovadoras y contemporáneas, con el nivel de los profesionales en el ámbito internacional.

\section{Referencias bibliográficas}

Acevedo-Duque, Á., Argüello, A. J., Pineda, B. G., y Turcios, P. W. (2020). Competencias del docente en educación online en tiempo de COVID-19: Universidades Públicas de Honduras. Revista de Ciencias Sociales (Ve), XXVI(E-2), 206224. https://doi.org/10.31876/rcs. v26i0.34123

Aledo, A (1995). El análisis de competencias 
¿Un cambio hacia el learning organization? Capital Humano, (81), 52-55.

Alles, M. (2008). Desarrollo del talento humano basado en competencias. Nueva edición actualizada. Editorial Granica.

Bedard, R. (2003). Los fundamentos del pensamiento y las prácticas administrativas: 1-El rombo y las cuatro dimensiones filosóficas. Revista Ad Minister, (3), 68-88.

Burns, R. W., y Klingstedt, J. L. (1973). Competency based education: An introduction. Englewood Cliffs, Education Technology Publication.

Cabrera, N., Cantelar, N., y Valcárcel, N., (2017). La formación por competencias de los profesionales del Instituto de Medicina Tropical "Pedro Kouri”. Revista Habanera de Ciencias Médicas, 16(6), 985-996.

Casanova, I., Canquiz, L., Paredes, Í., e Inciarte, A. (2018). Visión general del enfoque por competencias en Latinoamérica. Revista de Ciencias Sociales (Ve), XXIV(4), 114-125.

Castañeda, M. L. (2015). La enseñanzaaprendizaje por competencias. Revista de la Facultad de Derecho de México, 65(264), 243-265. http://dx.doi.org/10.22201/ fder.24488933e.2015.264.60310

Cejas, M. F., Rueda, M. J., Cayo, L. E., y Villa, L. C. (2019). Formación por competencias. Reto de la Educación Superior. Revista de Ciencias Sociales (Ve), XXV(1), 94-104.

Chomsky, N. (2014). El beneficio es lo que cuenta: Neoliberalismo y orden global. Planeta.

Companioni, O. L. (2015). El proceso de formación profesional desde el punto de vista complejo e histórico-cultural.
Revista Electrónica "Actualidades Investigativas en Educación”, 15(3), 1-23. http://dx.doi.org/10.15517/aie. v15i3.21041

Del Alcázar, J. P. (2020). Competencias universitarias del proyecto tuning América Latina. Mentinno, https:// blog.formaciongerencial.com/ competencias-universitarias-delproyecto-tuning-america-latina/

Garbanzo-Vargas, G. M. (2016). Desarrollo organizacional y los procesos de cambio en las instituciones educativas, un reto de la gestión de la educación. Revista Educación, 40(1), 67-87. http://dx.doi.org/10.15517/revedu. v40i1.22534

García, M. A. (2020). Efecto de la teoría contingencial, en el modelo de gestión de riesgo integral de los bancos panameños. Revista FAECO Sapiens, 3(2), 64-83. https://doi.org/10.48204/j. faeco.v $3 \mathrm{n} 2 \mathrm{a} 5$

Hernández, A. G. (2018). La formación en administración: Reflexiones para la construcción de un modelo educativo. Universidad y Empresa, 20(34), 9-52. https://doi.org/10.12804/revistas. urosario.edu.co/empresa/a.5001

Hernández, R., Fernández, C., y Baptista, M. D. P. (2014). Metodología de la Investigación. McGraw-Hill/ Interamericana Editores, S. A.

Icarte, G. A., y Labate, H. A. (2016). Metodología para la revisión y actualización de un diseño curricular de una carrera universitaria, incorporando conceptos de aprendizajes basados en competencias. Formación Universitaria, 9(2), 3-16. https://doi.org/10.4067/S071850062016000200002

Jiménez-Silva, W., Larrea-Altamirano, J., Navarrete-Fonseca, M., y CastroAyala, E. (2019). Emprendimientos 
innovadores a partir de competencias cognitivas en estudiantes universitarios. Revista Venezolana de Gerencia (RVG), 24(85), 397-420.

Lara, D. J., Rodríguez, P., y Del Canto, E. (2016). La gerencia en el contexto actual venezolano. Sapienza Organizacional, 3(6), 65-92.

Levy-Leboyer, C. (1997). Gestión de las competencias. Ediciones Gestión 2000 .

Llopart, X. (1997). La gestión de los recursos humanos en base a competencias. Análisis de competencias en empresas de auditoría (Tesis doctoral). Universitat da Barcelona (UAB), Barcelona, España.

Lombana, J., Cabezas L., Castrillón, J., y Zapata, A. (2014). Formación en competencias gerenciales. Una mirada desde los fundamentos filosóficos de la administración. Estudios Gerenciales, 30(132), 301-313. $\quad$ http://dx.doi. org/10.1016/j.estger.2014.01.017

Management Charter Initiative - MCI (1991). Occupational standards for managers: Assessment guidance. MCI

Marín-Idagarra, D. A. (2013). La conformación del currículo en Administración: Un estudio desde el isomorfismo institucional. Estudios Gerenciales, 29(129), 466-475. https://doi. org/10.1016/j.estger.2013.11.012

McClelland, D. C. (1973). Testing for competences rather than for intelligence. American Psychologist, 28, 1-14.

McLagan, P. A. (1997). Competencies: The next generation. Training \& Development, 51(5), 40-47.

Merino, M. (2020). Perfil profesional de la carrera de administración, en una universidad peruana. Revista Espacios. 41(30), 216-231.
Noreña, D. (2018). Caracterización del liderazgo efectivo según el rombo filosófico de Renée Bédard (Tesis de maestría). Universidad EAFIT, Medellín, Colombia.

Organización de las Naciones Unidas para la Educación, la Ciencia y la Cultura - UNESCO (2009). Conferencia mundial de Educación Superior 2009: Las nuevas dinámicas de la educación superior y de la investigación para el cambio social y el desarrollo UNESCO. https://pep.unc.edu.ar/ wp-content/uploads/sites/46/2017/04/ Declaracion conferencia Mundial de Educacion Superior 2009.pdf

Rabanal, R., Huamán, C. R., Murga, N. L., y Chauca, P. (2020). Desarrollo de competencias personales y sociales para la inserción laboral de egresados universitarios. Revista de Ciencias Sociales (Ve), XXVI(2), 250258. https://doi.org/10.31876/rcs. v26i2.32438

Ramos, E. (2016). El proceso de gestión de la calidad contextualizada en la formación del profesional de negocios internacionales (Tesis doctoral). Universidad Señor de Sipán, Chiclayo, Perú.

Schroder, H. (1989). Managerial competence: The key to excellence. Kendall/Hunt Publishing Company.

Segredo, A. M. (2016). Aproximación evolución, teorías, enfoques y características que han sustentado el desarrollo de las organizaciones. Revista Cubana de Salud Pública, 42(4), 585-595.

Sistema Nacional de Evaluación, Acreditación y Certificación de la Calidad Educativa - SINEACE (2012). Educación Superior en el Perú: Retos para el Aseguramiento de la Calidad. SINEACE. https://www2.congreso. gob.pe/sicr/cendocbib/con4 uibd.nsf/ 
Ramos Farroñán, Emma Verónica; Otero Gonzáles, Carlos Alberto; Heredia Llatas, Flor Delicia y Sotomayor Nunura, Gioconda Del Socorro Formación por competencias del profesional en administración: Desde un enfoque contingencial

0198694D6FE2A18D05257AF5005E 5B08/\$FILE/107070418-Educacionsuperior-en-el-Peru-Retos-para-elaseguramiento-de-la-calidad.pdf

Sistema Nacional de Formación para el Trabajo - SENA (2003). ABC de las competencias. Cartilla 1. División de Comunicaciones. https://repositorio.sena.edu.co/ bitstream/handle/11404/882/ a b c c o m p e t e n c i a s - 1 . pdf? sequence $=12$ \&isAllowed $=y$

Tobón, S. (2008). Formación basada en competencias: Pensamiento complejo, diseño curricular y didáctica. ECO Ediciones.

Useche, M. C., y Artigas, W. (2019).
Competencias de administración. Comparación de la perspectiva estudiantil-gerencial. Revista Venezolana de Gerencia (RVG), 23(1), 384-402.

Zabala, C., Marcano, N., y Chávez, S. (2013). Enfoques para el diseño de competencias e inserción de las TIC en Educación. REDHECS. Revista Electrónica de Humanidades, Educación y Comunicación Social, 14(8), 160-183.

Zwerg-Villegas, A. M. (22 de agosto de 2011). La definición de Administración depende de lo que uno espera de ella. Semana. https://www.semana.com/ la-definicion-administracion-dependeespera-ella/133760/ 